\title{
Optimal design of full disks with respect to mixed creep rupture time
}

\author{
K. Szuwalski \& A. Ustrzycka \\ Cracow University of Technology, Poland
}

\begin{abstract}
The mixed rupture theory to the optimization problem for the complex stress state is used. The problem of optimal shape for the rotating full disk with respect to mixed rupture time is investigated. The mathematical model of mixed creep rupture is described by the system of five partial differential equations. Difficulty of the problem results from two types of nonlinearities: geometrical connected with the use of the finite strain theory and physical - the material is described by the Norton's creep law, here generalized for true stresses and logarithmic strains. Additional time factor leads to subsequent complications. The parametric optimization describing the initial shape of the disk is applied. The obtained results are compared with the optimal disks with respect to ductile creep rupture time.

Keywords: mixed creep rupture, structural optimization, full disk.
\end{abstract}

\section{Introduction}

Structural elements working under creep conditions belong to the relatively new branches of structural optimisation, started in the seventieth years of the last century. The problem of time to rupture evaluation is of obvious relevance for various machine parts working under high temperature conditions. Theoretical modelling of long time strength appears to be important. A broad presentation of various objective functions with division on time-dependent and timeindependent, was given by Życzkowski [1]. To the latter group belong criteria connected with creep rupture. Most papers on optimal structural design are based on the brittle creep rupture theory proposed by Kachanov (small strain theory). It was due to its relative simplicity - possibility of rigidification theorem 
application. Optimal solutions with respect to brittle creep rupture often coincide with uniform strength structures.

In the work published by Hoff [2], the moment of failure of a bar under tension is defined as the one at which the cross-sectional area becomes zero as a result of quasiviscous flow. It was shown that the calculated results are in good agreement with the experimental data (Mentl [3]).

Applications of the ductile rupture theory, proposed by Hoff in optimization problems are rather scarce as it requires finite strain theory. For the first time it was used by Szuwalski for optimization of bars under nonuniform tension [4] and for optimisation of Mises truss [5]. Some problems of prismatic tension rods were discussed by Pedersen [6]. Such an approach introduces not only physical nonlinearities, connected with creep law (usually Norton's law), but geometrical, resulting from the finite strain theory, as well. Additional time factor leads to such complication, that till now, only few papers were devoted to optimization with respect to ductile rupture time. The optimal full disks with respect to ductile creep rupture time were found by Szuwalski [7], and first attempts for annular disks were made by Szuwalski and Ustrzycka [8].

The Hoff's concept has certain limitations. It predicts contrary to observations that creep does not result in damage of material. Also, his scheme does not explain fractures at small strains (brittle ruptures) and the change of character of rupture (from ductile to brittle).

Here, we suggest a theoretical model for creep deformation of the disk, which takes into account ductility and embrittlement of the material. We emphasize that the physics of such phenomena is very complex. Application of mixed rupture theory proposed by Kachanov [9] takes into account both: geometrical changes diminishing of transversal dimensions resulting from large strains (as in ductile rupture) and growth of microcracks (as in brittle rupture). Straight line b (Figure 1) corresponds to the purely brittle fracture and $d$ to the ductile fracture according to experimental data. The curve $\mathrm{m}$ showing relations for the mixed rupture, asymptotically approaches straight lines $\mathrm{b}$ and $\mathrm{d}$.

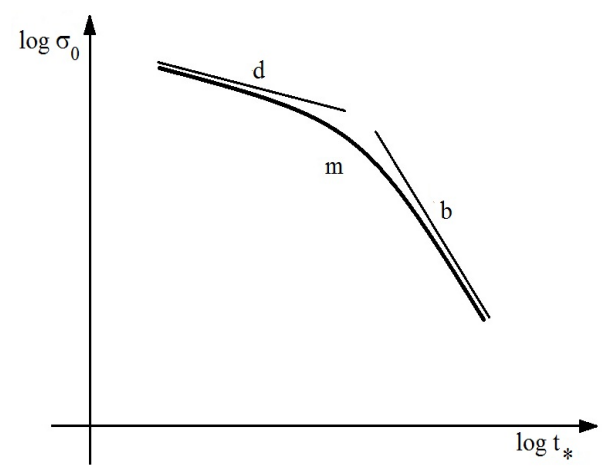

Figure 1: m- mixed rupture, d- Hoff's ductile creep rupture, b- Katchanov's brittle rupture. 
The first attempt of application the mixed rupture theory to shape optimization was made by Szuwalski and Ustrzycka [10] for bars under nonuniform tension.

In present paper the problem of optimal shape with respect to mixed creep rupture time for the complex stress state - rotating full disk is investigated. We assume that microcracking and diminishing of transversal dimensions start from the very beginning of creep process. Such an approach introduces not only physical nonlinearities, connected with creep law (usually Norton's law), but geometrical, resulting from finite strain theory, as well. Additional time factor leads to subsequent complications. The whole creep process must be analyzed from its beginning up to rupture.

\section{Governing equations}

The concept of the mathematical description of mixed creep rupture requires an examination of the entire process, taking into account geometrical changes occurring in the course of it. The problem is solved in material (Lagrangean) coordinates and all parameters in initial state, for time equal zero, are denoted by capital letters, while current values of these parameters by the same small letters. Due to axial symmetry all quantities will be functions of two independent variables: radius $\mathrm{R}$ and time $\mathrm{t}$. The disk rotates with constant angular velocity $\omega$ and body forces connected with own mass of the disk are taken into account (Figure 2).

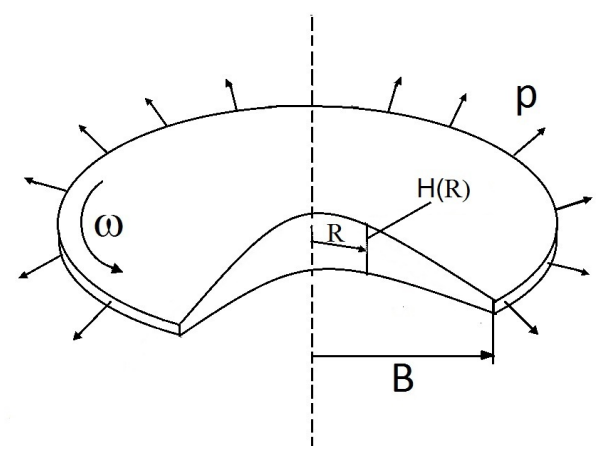

Figure 2: Model of the rotating full disk.

The material of the disk fulfills the Norton's creep low:

$$
\dot{\varepsilon}_{e}=k \sigma_{e}^{n}
$$

where $\sigma_{e}$ denotes the effective stress, according to the Huber-Mises-Hencky hypothesis generalized to true stresses:

$$
\sigma_{e}=\left(\sigma_{r}^{2}+\sigma_{\vartheta}^{2}-\sigma_{r} \sigma_{\vartheta}\right)^{\frac{1}{2}}
$$


and respectively, $\dot{\varepsilon}_{e}$ is the effective strain rate, $\mathrm{k}$ and $\mathrm{n}$ are material constants.

The internal equilibrium condition for plane stress state, with body force, takes form:

$$
\frac{1}{h r^{\prime}} \frac{\partial}{\partial R}\left(h \sigma_{r}\right)+\frac{\sigma_{r}-\sigma_{\vartheta}}{r}+\frac{\gamma}{g} \omega^{2} r=0
$$

where $\sigma_{r}$ stands for current value of radial stress and $\sigma_{\vartheta}$ of circumferential one, $\mathrm{h}$ - for current thickness, $\boldsymbol{\gamma}$ - specific weight of material and $\mathrm{g}$ - acceleration of gravity.

Assumption of incompressibility leads to:

$$
H R d R=h r d r
$$

where $\mathrm{R}$ stands for material coordinate of the discussed point, while $\mathrm{r}$ for spatial one.

Finite strains require logarithmic strains:

$$
\varepsilon_{r}=\ln \frac{\partial r}{\partial R}=\ln r^{\prime} ; \varepsilon_{\vartheta}=\ln \frac{r}{R} ; \varepsilon_{z}=\ln \frac{h}{H}
$$

The shape change law, assumed in form of similarity of true stresses and velocities of logarithmic strains deviators according to Szuwalski [7] leads to:

$$
\frac{\dot{r}}{r}=\frac{1}{2} k \sigma_{e}^{n-1}\left(2 \sigma_{\vartheta}-\sigma_{r}\right)
$$

Compatibility condition, after some rearrangements, presented by Szuwalski [7], takes form:

$$
\begin{aligned}
& \sigma_{r}^{\prime}\left[(n-1)\left(2 \sigma_{r}-\sigma_{\vartheta}\right)\left(2 \sigma_{\vartheta}-\sigma_{r}\right)-2 \sigma_{e}^{2}\right]+\sigma_{\vartheta}^{\prime}\left[(n-1)\left(2 \sigma_{\vartheta}-\sigma_{r}\right)^{2}+4 \sigma_{e}^{4}\right]= \\
& =6 \sigma_{e}^{2} \frac{r^{\prime}}{r}\left(\sigma_{r}-\sigma_{\vartheta}\right) .
\end{aligned}
$$

To find the mixed rupture time, the evolution equation proposed by Kachanov [9] will be applied:

$$
\frac{\partial \Psi}{\partial t}=-D\left[\frac{\sigma_{e}}{\Psi}\right]^{m}
$$

in which $D$ and $m$ are material constants.

Continuity function $\Psi$ is defined by the ratio of effective cross - sectional area $a_{e f}$ to undamaged area $a$ :

$$
\Psi=\frac{a_{e f}}{a}
$$

In contrast with brittle rupture theory $\sigma$ denotes here the true stress - related to the current cross - section $a$ (geometrical changes are taken into account). 
We characterize damage by the continuity function $0 \leq \Psi \leq 1$. At the initial moment (no damage): $\Psi=1$, as time goes on, it decreases. The moment of rupture corresponds to a value $\Psi=0$ at which fracture localizes.

We define the rupture criterion in the following form:

$$
\exists R:\left(\left.R \in\langle 0, l\rangle \wedge \Psi \in\langle 1,0\rangle \wedge \Psi(\sigma)\right|_{t_{*}^{(m)}} \rightarrow 0\right)
$$

Time after which the continuity function will diminish to zero will be the time of mixed rupture $t_{*}^{(m)}$.

For the sake of numerical calculations, dimensionless quantities are introduced. Both material and spatial coordinates are related to the initial external radius $B$ :

$$
\hat{R}=\frac{R}{B} ; \hat{r}=\frac{r}{B}
$$

The thickness of the disk is related to the mean thickness $h_{m}$ of the full disk of volume $\mathrm{V}$ and radius $\mathrm{B}$ :

$$
\hat{H}=\frac{\pi B^{2}}{V} \cdot H ; \hat{h}=\frac{\pi B^{2}}{V} \cdot h
$$

Radial loading at radius $b$ of the rotating disk is resulting from mass $M$ uniformly distributed on the outer edge.

$$
\sigma_{r}(b)=p=\frac{M \omega^{2}}{2 \pi h(B)}
$$

Dimensionless stresses are referred to stresses calculated using a rigidification theorem in the motionless full plane disk subject to tension with uniform pressure $p(13)$ :

$$
\hat{\sigma}_{i}=\frac{2 V}{M \omega^{2} B^{2}} \sigma_{i} ; i=r, \vartheta
$$

Consequently dimensionless time is defined:

$$
\bar{t}=\frac{t}{t^{(d)}}
$$

where: $t^{(d)}$ stands for the time of ductile rupture for full plane disk (Szuwalski [7]). To avoid the large number of material constants in numerical calculations we introduce the new parameter $\Theta$. This parameter is equal to the radio of the brittle rupture time to the ductile rupture time for the prismatic bar subject to tension with the initial stress $\sigma_{s}$ :

$$
\Theta=\frac{t_{p r}^{(K)}}{t_{p r}^{(H)}}=\frac{n k \sigma_{s}^{n}}{(m+1) D \sigma_{s}^{m}}
$$


where $\sigma_{s}$ is equal:

$$
\sigma_{s}=\frac{M \omega^{2} R_{0}^{2}}{2 V}
$$

The parameter $\Theta$ contains four material constants: $n$ and $k$ from Norton's law (1) and $m$ and $D$ from evolution equation (8). In some way it describes sensitivity of material on type of damage: brittle or ductile.

The mathematical model of mixed creep rupture is described by the system of five partial differential equations in dimensionless form:

$$
\begin{gathered}
\hat{\sigma}_{r}^{\prime}=\frac{\hat{r}^{\prime}}{\hat{r}}\left(\hat{\sigma}_{r}-\hat{\sigma}_{\vartheta}\right)-2 \cdot \hat{r} \hat{r}^{\prime} \mu-\frac{\hat{h}^{\prime}}{\hat{h}} \hat{\sigma}_{r} \\
\hat{\sigma}_{\vartheta}^{\prime}=\frac{6 \hat{\sigma}_{e}^{2}\left(\hat{\sigma}_{r}-\hat{\sigma}_{\vartheta}\right) \frac{\hat{r}^{\prime}}{\hat{r}}-\hat{\sigma}_{r}^{\prime}\left[(n-1)\left(5 \hat{\sigma}_{r} \hat{\sigma}_{\vartheta}-2 \hat{\sigma}_{r}^{2}-2 \hat{\sigma}_{\vartheta}^{2}\right)-2 \hat{\sigma}_{e}^{2}\right]}{(n-1)\left(2 \hat{\sigma}_{\vartheta}-\hat{\sigma}_{r}\right)^{2}+4 \hat{\sigma}_{e}^{2}} \\
\frac{d \hat{r}}{d \hat{t}}=\frac{\hat{r}}{2 \cdot n}\left(\hat{\sigma}_{r}^{2}+\hat{\sigma}_{\vartheta}^{2}-\hat{\sigma}_{r} \hat{\sigma}_{\vartheta}\right)^{\frac{n-1}{2}}\left(2 \hat{\sigma}_{\vartheta}-\hat{\sigma}_{r}\right) \\
\frac{\partial \Psi}{h \hat{H} \hat{R}}=\frac{-1}{\hat{r}^{\prime} \hat{r}}\left[\frac{\hat{\sigma}_{e}}{\Psi}\right]^{m} .
\end{gathered}
$$

In presented above equations we have five unknowns: true stresses $\sigma_{r}$ and $\sigma_{\vartheta}$, current thickness $-\mathrm{h}$, spatial radial coordinate $-\mathrm{r}$ and continuity function $\Psi$.

At the initial moment $(t=0)$ disk remains undeformed, therefore the initial conditions take form:

$$
\hat{r}(\hat{R}, 0)=\hat{R} ; \hat{h}(\hat{R}, 0)=\hat{H}(\hat{R})
$$

The boundary conditions are described in this form:

$$
\begin{gathered}
\hat{r}(0, \hat{t})=0 ; \quad \dot{\hat{r}}(0, \hat{t})=0 \\
\hat{\sigma}_{r}(0, \hat{t})=\hat{\sigma}_{\vartheta}(0, \hat{t})
\end{gathered}
$$

The condition at external radius, where the mass $M$ is distributed, in dimensionless form may be written:

$$
\hat{\sigma}_{r}(1, t)=\frac{1}{\hat{h}(1, t)}
$$


In the fourth of equations (16) and in the second of initial conditions (17), we have the function $\hat{H}(\hat{R})$ describing the initial profile of the disk. It is necessary to know this function in order to solve the set (16). Because this function is being sought in the optimisation process, we shall apply parametric optimisation. We shall look for the best possible function $\hat{H}(\hat{R})$, leading to the longest lifetime to mixed rupture in assumed class of polynomial functions.

Let's consider an optimality criterion in the form:

$$
(\exists ! \hat{H}(\hat{R}) \in f) \quad t_{*}^{(m)} \underset{V=\text { const. }}{\longrightarrow} \max
$$

where functions $f: \mathrm{R} \rightarrow \mathrm{R}, \quad R \rightarrow b_{0}+b_{1} R+b_{2} R^{2}+\ldots+b_{l} R^{i}, \quad$ for all arguments $R \in\langle 0,1\rangle$, where $i \in \mathrm{N} \subset\{0\} \quad$ ( $i$ is a non-negative integer) and $b_{l} \in \mathrm{R}$ are constant coefficients, where $l=0,1,2, \ldots, i$, and $b_{l} \neq 0$.

\section{Numerical algorithm}

In order to perform an optimisation procedure, the rupture and optimality criterion to the mathematical model is introduced. The numerical algorithm is proposed for the complex stress state. We must follow step by step the whole creep process for each new geometry of the deformed disk up to the moment of fulfilling of rupture criterion in order to establish the mixed creep rupture time. The numerical algorithm consists of two blocks, which are sequentially activated.

The algorithm begins by defining the initial geometry, constant parameters and the boundary conditions for the stresses (20). In the first block, for given geometry of the disk, the true stresses distribution is established by integration of the first and the second equations of the set (18) with respect material coordinate $\hat{R}$. The Runge - Kutta fourth order method is applied. We do not know values of stresses in the centre of the disk, so they are assumed arbitrarily, but they must satisfy the boundary condition at the outer edge of the disk (21). Therefore, the recurrential procedure must be applied. Found distribution of true stresses, with help of the evolution equation (the last from the set (18) makes it possible to establish the distribution of continuity function $\Psi$. If it's minimal value satisfies the rupture criterion, the creep process finishing - the time to mixed rupture was found.

When values of continuity function are sufficiently large, the new changed geometry of the disk is calculated. The integration of the third of equation (18) with respect to time is performed using Euler's method. The time dependent solutions have high sensitivity on time step dimension, so we choose the varying time step, depending on current creep velocity. In this way new spatial coordinates are calculated. Finally incompressibility condition (fourth of set (18)) makes it possible to find changed shape (thickness) of the disk. From the results obtained for many initial shapes of the disk, described by assumed polynomial function, the best leading to the longest time to mixed creep rupture 
is chosen - this is the optimal disk. Consequently, for the new geometry of the disk, the full cycle of the above mentioned calculations are repeated.

\section{Discussion of results}

At the beginning optimal solutions for the problem of rotating full disk with respect to mixed creep rupture time are sought in the class of linear functions:

$$
\hat{H}\left(\hat{R} ; u_{0}, u_{1}\right)=u_{0}+u_{1} \hat{R}
$$

Parameters $u_{0}$ and $u_{1}$ (uniparametric optimisation), which optimal values are sought, are linked together by the condition of given volume $\mathrm{V}$ :

$$
u_{1}=\frac{3}{2} \frac{u_{0}\left(\beta^{2}-1\right)+1}{\left(1-\beta^{3}\right)}
$$

The influence of parameter $\mu$ as the ratio of own mass of the disk to mass uniformly distributed at the outer edge and the parameter $\Theta$ ratio of the brittle rupture time and ductile rupture time of the prismatic bar, rotating with the constant angular velocity $\omega$, loaded by mass $M$ distributed at the external edge, with the bar's own mass neglected is investigated. Profiles of optimal disks for uniparametric optimization are shown in Figure 3 as a function of the parameter $\mu$ for three different values of parameter $\Theta$

Obtained solutions strongly depend on ratio $\Theta$ and $\mu$. When the mass $M$ is very large in comparison with own mass of the disk (small values of $\mu$ ) optimal disks are close to flat ones. For the larger values of parameter $\Theta$ (ductile materials) the thickness of optimal disks in the vicinity of outer edge grows. For larger values of parameters $\mu$ (small mass $M$ at the outer radius), the mass of the disk is distributed as close to the rotation axis as possible.

Better results may be obtained for disks, which initial shape is defined by quadratic function:

$$
\hat{H}\left(\hat{R} ; b_{0}, b_{1}, b_{2}\right)=b_{0}+b_{1} \hat{R}+b_{2} \hat{R}^{2}, b_{2} \neq 0
$$

Because now we have three parameters, finding of their optimal values takes much more time than for uniparametric optimization. From three parameters in this function, only two may be treated as free ones, the third results from given volume of disk:

$$
\hat{V}=\int_{0}^{1} 2 \pi\left(b_{0}+b_{1} \hat{R}+b_{2} \hat{R}^{2}\right) \hat{R} d \hat{R}=\pi
$$

from which:

$$
b_{2}=2-2 b_{0}-\frac{4}{3} b_{1} .
$$

Profiles of optimal disks for biparametric optimization are shown in Figure 4. 
A) $\Theta=0,4$

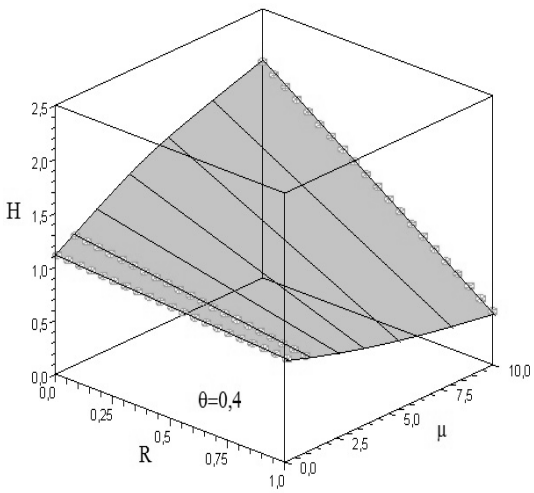

B) $\Theta=3$

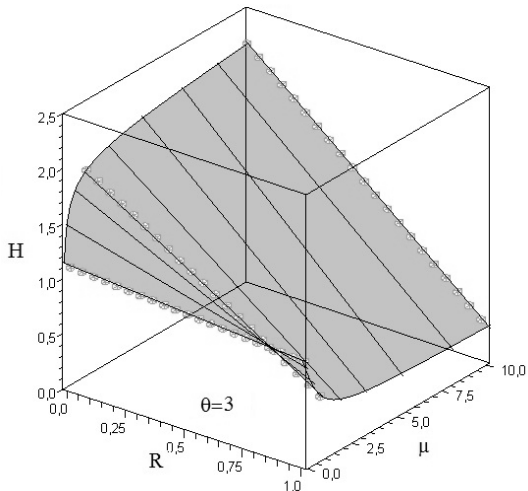

Figure 3: Optimal shapes of the disks for uniparametric optimisation.

A) $\Theta=0,4$

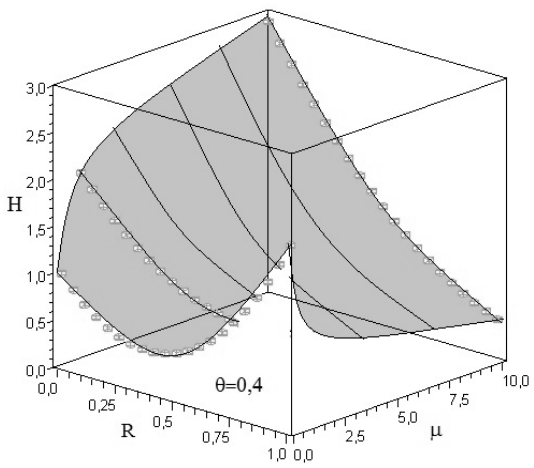

B) $\Theta=3$

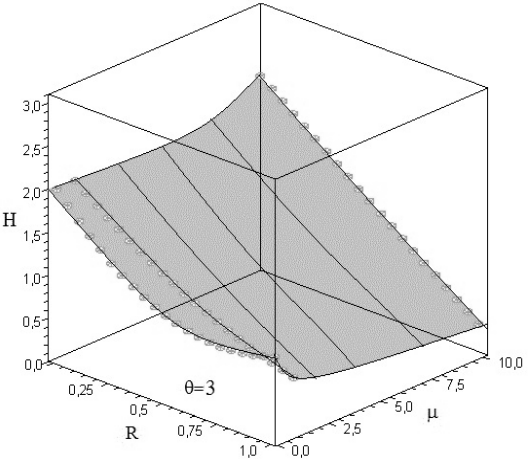

Figure 4: Optimal shapes of the disks for biparametric optimisation.

For smaller values of parameters $\mu$ (the own mass almost neglected), the growth of thickness at the outer edge was observed, optimal solutions have minimum inside the disk width. The larger thickness at the outer edge works as some kind of reinforcement and thanks to it time to mixed rupture may be longer. For larger values of parameters $\Theta$ (brittle materials), this effect is smaller.

In Figure 5A) the changed profiles of the optimal disk and in Figure 5B) the corresponding distribution of continuity function at the same time moments are shown. The results are presented for optimal disk for parameters: $\mu=0.1, n=3, m=2, \Theta=3$, which initial shape is described by function $H(R)=2-3 R+2 R^{2}$.

We observed that despite the strengthening of the outer edge of the disk, the rupture criterion for the continuity function $\Psi$ is fulfilled there. Inside the disk the values of function $\Psi$ are quite large. This effect is due to limitation only to the disks of initial profile described by quadratic function (23). 
A)

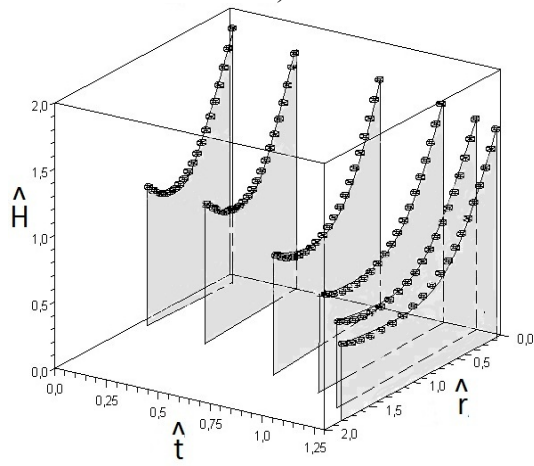

B)

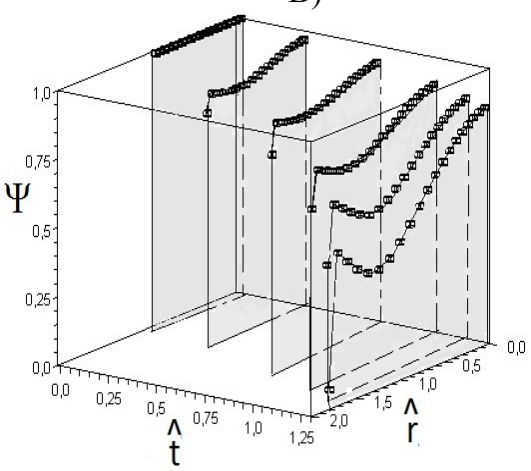

Figure 5: Time cross-section of creep process.

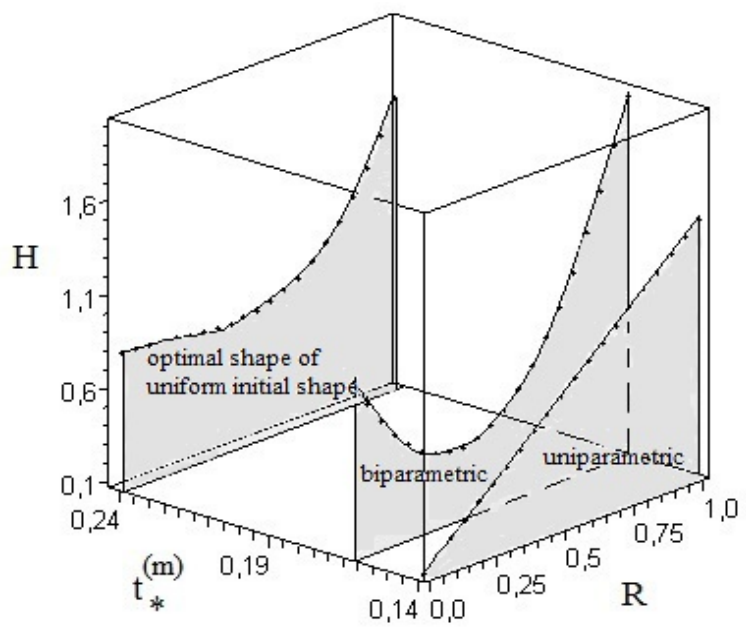

Figure 6: Optimal shape of the uniform initial strength disks compared with uni- and biparametric optimisation.

We may expect that disks of uniform initial strength, in which both radial and circumferential stresses are the same for $0 \leq R \leq B$ are close to optimal with respect to mixed rupture time.

Such disks described by formula:

$$
\hat{H}_{u s}(\hat{R})=\frac{1}{\hat{\Sigma}} \exp \left[\frac{\mu}{\hat{\Sigma}}\left(1-\hat{R}^{2}\right)\right],
$$

where: $\hat{\Sigma}$ - dimensionless equalized initial stress, calculated from the condition of constant volume:

$$
\hat{\Sigma}=\frac{\mu}{\ln (1+\mu)},
$$


may be slightly corrected, to obtain the longest life - time to creep rupture. Correction is adopted in form of the third degree polynomial function:

$$
\hat{H}_{c o r}=p_{0}+p_{2} \hat{R}^{2}+p_{3} \hat{R}^{3},
$$

without the linear element, so as to the thickness derivative in the middle of the disk was equal to zero.

As the correction cannot change the total volume of the bar, only two coefficients of eq. (30) may be treated as free parameters, while the third results from the constant volume condition:

$$
p_{3}=-\frac{5}{2} p_{0}-\frac{5}{4} p_{2} .
$$

For different values of these parameters we designated the initial shape of the disk,

$$
\hat{H}(\hat{R})=\hat{H}_{u s}(\hat{R})+\hat{H}_{\text {cor }}(\hat{R})
$$

then we integrate the system of equations (16)

Calculations were carried for $\mu=0,1, \Theta=3$, exponent in Norton's law $n=3$ and exponent in Kachanov's law $m=2$. Optimal shapes of the corrected shape of uniform initial strength the disk compared with obtained earlier for parametric optimization are presented in Figure 6.

The optimal shapes of different disks are placed on the time axis at the points corresponding to obtained times $m$ of mixed rupture. As expected, the corrected shape of uniform initial strength disk provides the longest time to mixed creep rupture. Introduction of parabolic disk enlarges this time a for about $14 \%$, while for corrected disk of uniform strength the gain in comparison with conical disk is about $70 \%$.

\section{Conclusions}

In conclusion, it is worth pointing out that the mathematical model of mixed creep rupture discussed in this paper addresses one of the fundamental phenomena that occur at high temperatures in the materials. The theoretical model for creep deformation of the disk with the account of ductility and embrittlement of the material is described. Application of mixed rupture theory proposed by Kachanov in this model takes into account: geometrical changes diminishing of transversal dimensions resulting from large strains (as in ductile rupture) and growth of microcracks (as in brittle rupture). The time to rupture is defined as a time when continuity function $\Psi$ diminishes to zero.

The paper is embedded in the branch of research dedicated to optimum design of structures under creep conditions with the objective function in the form of mixed creep rupture time. The problem of optimal shape with respect to mixed creep rupture time for the complex stress state - rotating full disk is investigated. Both the own mass of the disk and a mass uniformly distributed on the outer 
radius are taken into account. The Norton creep law has been applied. The problem has been solved in the framework of large strains - true stresses approach. The logarithmic measure of strains has been adopted. The optimal profiles of disks, satisfying the condition of maximum time to rupture, were found for full disks and for the constant volume of material. The best profile of the disk, leading to the longest lifetime to mixed rupture in assumed class of polynomial functions was sought.

The corrected shape of uniform initial strength disk provides the longest time to mixed creep rupture. Correction is adopted in form of the third degree polynomial function. In comparison of conical disk enlarges the time to mixed rupture for about $70 \%$.

The results presented in the paper have fundamental meaning for possible applications in different technologies. Both the power plants or the automobile industry and ship or aircraft technologies can profit from decent analysis presented in the submitted manuscript. Also, this domain of research looks promising for the future studies in view of multi-scale modeling applied to creep rupture mechanisms.

\section{References}

[1] Życzkowski M., Optimal structural design under creep conditions, Appl. Mech. Rev. 12, 453 - 461, 1988.

[2] Hoff N.J., The necking and rupture of rods subjected to constant tensile loads, J. Appl. Mech. Trans. ASME 20 105-112, 1953.

[3] Mentl V., An application of a phenomenological theory of creep damage, Materials at High Temperatures, 23, 195-200, 2006.

[4] Szuwalski K., Optimal design of bars under nonuniform tension with respect to ductile creep rupture, Mech. Struct. Mach. 3, 303-319, 1989.

[5] Szuwalski K., Optimal design of Mises truss with respect to time to creep rupture, Engng. Trans. 42, 145-155, 1994.

[6] Pedersen, P., On the influence of boundary conditions, Poisson's ratio and material non-linearity on the optimal shape, Int. J. of Solids and Structures 38(3), 465-477, 2001.

[7] Szuwalski K., Optimal design of disks with respect to ductile creep rupture time, Strict. Opt. 10, 54-60, 1995.

[8] Szuwalski K., Ustrzycka A., The Influence of Boundary Conditions on Optimal Shape of Annular Disk With Respect to Ductile Creep Rupture Time, European Journal of Mechanics, (2012), (in print).

[9] Kachanov L.M., Creep theory, Fizmatgiz, Moskwa, 1960.

[10] Szuwalski K., Ustrzycka A., Optimal Design of Bars Under Nonuniform Tension With Respect to Mixed Creep Rupture Time, International Journal of non-linear mechanics, 2012, (in print). 